\title{
Adaptive Precoding for Wireless MIMO Broadcast Channels with Limited Feedback*
}

\author{
James (Sungjin) KIM ${ }^{\dagger, \dagger \dagger a)}$, Student Member, Hojin KIM ${ }^{\dagger \dagger}$, Chang Soon PARK ${ }^{\dagger}$, \\ and Kwang Bok LEE ${ }^{\dagger}$, Nonmembers
}

\begin{abstract}
SUMMARY Recently, a number of techniques have been introduced to exploit multiuser diversity of a wireless multiple-input multiple-output (MIMO) broadcast channel (BC) that consists of a base station with $t$ transmit antennas and $K$ users with multiple antennas. However, prior works have ignored the rate overhead associated with feedback of MIMO BC channel state information at transmitter (CSIT), which is roughly $K$ times larger than single-user MIMO CSIT (i.e., it is $O(t r)$ where $r=\sum_{k=1}^{K} r_{k}$ and $r_{k}$ is the number of antennas at the $k$ th user). Considering the amount of feedback signaling, quantization is a necessity for effective feedback transmission as a form of partial CSIT. In this paper, we propose the greedy multi-channel selection diversity (greedy MCSD) scheme based on block MMSE QR decomposition with dirty paper coding (block MMSE-DP), where partial CSIT is almost sufficient. The sum-rate performance of our novel scheme approaches extremely close to the sum capacity of MIMO $\mathrm{BC}$ as the number of users increases, whereas the feedback overhead is reduced by a factor of $2 t^{3} / L\left(t^{2}-t\right)$, in which $L$ is the number of active channel vectors. Simulation results validate the expectation from the analysis. In addition, the proposed scheme is shown to be appropriate for reconfigurable implementation.

key words: MIMO, broadcast channel (BC), adaptive precoding, SDR implementation
\end{abstract}

\section{Introduction}

Multiple-input multiple-output (MIMO) systems have been one of key techniques to achieve high rate and high reliability over wireless broadcast channels [2]-[6]. The investigation of the capacity region has been of concern in an MIMO broadcast channel (BC), where the base station has multiple transmit antennas and each user has possibly multiple receive antennas [7]-[13]. In [8], it was shown that an achievable rate region for the multiple-input singleoutput (MISO) BC is obtained by applying dirty paper coding (DPC), or known interference cancellation, at the transmitter [14]. Sum-power iterative water-filling (SP-IWF) provides the optimum transmission policies, whereas reducing the computational complexity and feedback overhead is still an ongoing research area [13], [15]. As a low computational complexity approach, the greedy-type user selection [16] and the joint-channel decomposition [9], [17], [18] are utilized instead of the optimal power allocation pol-

\footnotetext{
Manuscript received March 9, 2005.

Manuscript revised May 31, 2005.

${ }^{\dagger}$ The authors are with the EECS/EE at Seoul National University, Seoul, Korea.

${ }^{\dagger \dagger}$ The authors are with the Commun. \& Network Lab at SAMSUNG AIT, Suwon, Korea.

${ }^{*}$ This paper was presented in part at SPAWC 2005 [1].

a)E-mail: kimsj@mobile.snu.ac.kr

DOI: 10.1093/ietcom/e88-b.11.4237
}

icy, respectively. For feedback reduction, the random beamforming technique, or opportunistic beamforming [19], for MIMO BC is introduced in [20], where a significant number of users is, however, required to achieve sum capacity compared to the case of deterministic beamforming. As another approach for feedback reduction, the efficient vector quantization [21] is developed to represent the partial channel state information at the transmitter (CSIT) [22], where, however, most attention has been focused on single-user MIMO systems. That is, prior works on sum-rate near-optimal transmit schemes do not take into account the feedback overhead, to our best knowledge.

In this paper, we propose the cost-effective scheme in terms of complexity and feedback overhead that obtains near the maximum sum-rate of the wireless MIMO BC. As a solution to this problem, we propose the greedy multi-channel selection diversity (greedy MCSD) scheme based on novel block MMSE QR decomposition with DPC (block MMSEDP) where DP is an abbreviation for dirty paper. Simulation results indicate that the sum-rate performance of our scheme approaches extremely close to the sum capacity of MIMO BC with only a few users (e.g., a gap of $0.4 \mathrm{bps} / \mathrm{Hz}$ from SP-IWF), whereas the feedback overhead is significantly reduced.

The remainder of this paper is organized as follows. In Sect. 2, the system model is introduced. In Sect. 3, SPIWF, greedy-type user selection, and time-division multipleaccess MIMO (TDMA-MIMO) are analyzed. Our novel scheme is presented in Sect. 4. Section 5 provides the analysis of sum-rate performance. Numerical results are presented in Sect. 6 and reconfigurable implementation using the software defined radio technology is discussed in Sect. 7. Finally, we conclude in Sect. 8.

Notation: the superscripts ${ }^{T}$ and ${ }^{H}$ stand for transpose, conjugate transpose, respectively. The cardinality of the set $S$ is notated as $|S|$.

\section{System Model}

Consider a $K$ user wireless communication system with multiple transmit antennas at the base station and multiple receive antennas for each user, as shown in Fig. 1. We assume that the base station has $t$ transmit antennas, user $k$ has $r_{k}$ receive antennas, and the number of all receive antennas in the system is $r=\sum_{k=1}^{K} r_{k}$. Also, we model the channel as a frequency-flat block fading channel. Interference from 


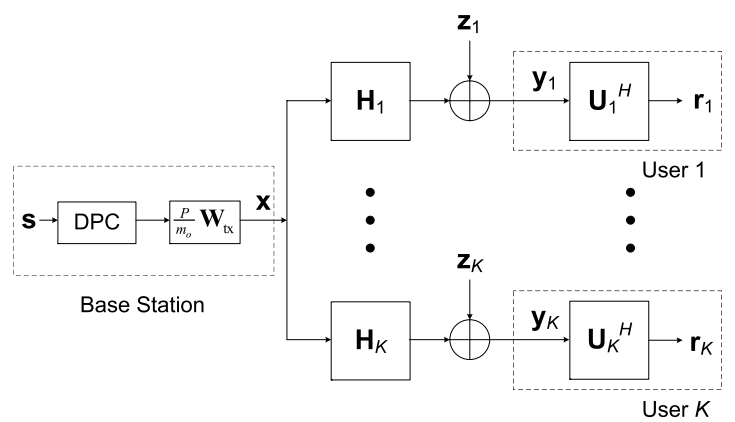

Fig. 1 Greedy MCSD with block MMSE-DP in a MIMO broadcast channel.

neighboring cells is modeled as additive Gaussian noise, as we concentrate on the single cell model. The received signal of user $k$ is expressed as

$$
\mathbf{y}_{k}=\mathbf{H}_{k} \mathbf{x}+\mathbf{z}_{k}
$$

where $\mathbf{x}$ is the $t \times 1$ sum transmit signal vector, i.e., $\mathbf{x}=$ $\sum_{k=1}^{K} \mathbf{x}_{k}$ in which $\mathbf{x}_{k}$ is the transmit signal vector intended for user $k$. The total sum transmit power of all users is constrained by $P$, i.e., $\operatorname{tr}\left(\sum_{k=1}^{K} \boldsymbol{\Sigma}_{k}\right) \leq P$ where $\boldsymbol{\Sigma}_{k}=E\left[\mathbf{x}_{k} \mathbf{x}_{k}^{H}\right]$ is the transmit covariance matrix of user $k$. The $r_{k} \times 1$ vector $\mathbf{z}_{k}$ represents the random additive noise for user $k$ where $\mathbf{z}_{k} \sim \mathcal{C N}(\mathbf{0}, \mathbf{I})$. The channel $\mathbf{H}_{k}$ is an $r_{k} \times t$ matrix, whose entries are assumed to be independent and identically distributed (i.i.d.) circularly symmetric complex Gaussian random variables with zero-mean and unit variance. Also, $\mathbf{H}_{k}$ is independent of $\mathbf{H}_{j}$ for all $j \neq k$.

In general, it is difficult for the base station to have the perfect knowledge of CSIT because the feedback link has delayed lossy feedback characteristics. Hence, the problem at hand is to find the transmit and receive structure that minimizes the feedback rate subject to the performance constraint such that the data throughput is kept as close as possible to the sum capacity.

\section{SP-IWF, TDMA-MIMO, and MMSE-DP}

In this section, we describe the following approaches: SPIWF [13], TDMA-MIMO [23], and greedy MMSE-DP. The sum-rate maximization can be solved by using SP-IWF, which achieves the sum capacity of an MIMO BC at the cost of higher complexity. In the case of TDMA-MIMO, the base station transmits to only a single-user at a time by using all transmit antennas, which is a suboptimal solution when the base station has multiple transmit antennas. So the optimality holds if and only if the number of transmit antennas is equal to one. It is shown that the maximum sum-rate of TDMA-MIMO is the largest single-user capacity of the $K$ users, which is given by

$$
C_{\mathrm{TD}} \triangleq \max _{i=1, \ldots, K} C\left(\mathbf{H}_{i}, P\right)
$$

where $C\left(\mathbf{H}_{i}, P\right)$ denotes the single-user capacity of the $i$ th user subject to power constraint $P$.
For the comparison purpose, we present the algorithm of greedy MMSE-DP for MIMO, which is the updated version of greedy (ZF) QR decomposition with DPC (ZF-DP) for MISO in [16] so as to apply MMSE filtering and to consider the receivers equipped with multiple receive antennas. In this case, the receive array gain is, however, not exploited (see Lemma 2). The algorithm for greedy MMSE-DP is outlined in Appendix.

\section{Greedy MCSD with Block MMSE-DP}

We propose a multiuser MIMO scheme, i.e., greedy MCSD with block MMSE-DP, on the basis of the transmit structure in per-user unitary $\mathrm{FB} / \mathrm{BF}$ and rate control $\left(\mathrm{PU}^{2} \mathrm{RC}\right)$ described in [24]. FB and BF abbreviate feedback and beamforming, respectively. The transmitter structure is shown in Fig. 2, where the precoding block, consisting of DPC and beamforming, is employed at the transmitter. Beamforming is performed using unitary matrix $\mathbf{W}_{\mathrm{tx}}$ that is a function of unitary matrix $\mathbf{V}_{k}$ and diagonal matrix $\mathbf{D}_{k}$ for all $k$, which are obtained by singular value decomposition (SVD) such that $\mathbf{H}_{k}=\mathbf{U}_{k} \mathbf{D}_{k} \mathbf{V}_{k}^{H}$. Using space division multiple access (SDMA) approach, the data stream of each user can be allocated to each beam vector of the unitary transform matrix $\mathbf{W}_{\mathrm{tx}}$ depending on channel conditions. Also, the transmitter adjusts the data rate per-stream independently. Note that by this structure, the proposed scheme uses spatial multiplexing to transmit simultaneously to multiple users, and multiple streams are transmitted to multiple users.

In [24], it was proposed that $\mathrm{PU}^{2} \mathrm{RC}$ performs unitary beamforming with a finite set in a predetermined way, where combining with the user and beam selection leads to additional throughput improvement due to interference reduction between users. The amount of feedback information can be reduced by using a predetermined finite set, e.g., applying a codebook design such as Grassmannian line packing to the finite set [25]. However, performance is severely degraded when there are smaller number of receive antennas than transmit antennas. To mitigate the performance degradation for such a case, in this paper we propse the enhanced $\mathrm{PU}^{2} \mathrm{RC}$ scheme, i.e., greedy MCSD with Block MMSE-DP, that is effectively combined with DPC. A detailed discussion of this scheme will be found in the following subsections. In brief, our proposed scheme uses known interference cancellation and non-predetermined beamforming at the transmitter.

\subsection{Block MMSE-DP}

This section discusses block MMSE QR decomposition, noting that the combination of block MMSE QR decomposition with known interference cancellation, or DPC, is denoted as block MMSE-DP throughout this paper. As the first stage of block QR decomposition, the channel is rotated using the left unitary matrix $\mathbf{U}_{k}$ obtained by SVD of the each user channel (i.e. $\mathbf{H}_{k}=\mathbf{U}_{k} \mathbf{D}_{k} \mathbf{V}_{k}^{H}$ ). This is equivalent to the process that sets the receiver spatial filtering as $\mathbf{W}_{\mathrm{rx}}=\mathbf{U}_{k}^{H}$ 


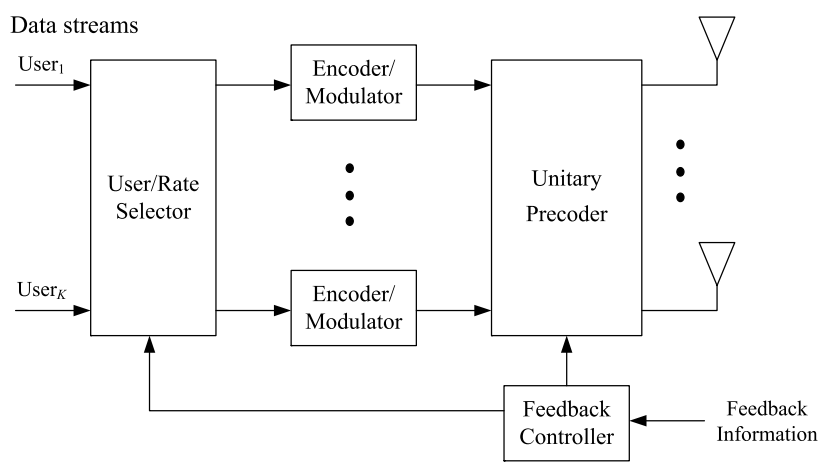

Fig. 2 Transmitter of the greedy MCSD with block MMSE-DP scheme.

(see Lemma 1). By feeding back $\mathbf{F}_{k}$ instead of $\mathbf{H}_{k}$ to the base station, the feedback overhead can be reduced by a factor of $2 t^{2} /\left(t^{2}-t\right)$ as described in [22], where the rotated channel matrix $\mathbf{F}_{k}$ is given by

$$
\mathbf{F}_{k}=\mathbf{U}_{k}^{H} \mathbf{H}_{k}=\mathbf{D}_{k} \mathbf{V}_{k}^{H} \text {. }
$$

It can be seen that rotating with the left unitary matrix decomposes MIMO channels into multiple MISO channels $\mathbf{F}^{H}=\left[\mathbf{F}_{1}^{H}, \ldots, \mathbf{F}_{K}^{H}\right]=\left[\mathbf{f}_{1}, \ldots, \mathbf{f}_{r}\right]$, which is referred to as the rotated channel matrix. We also denote each row of $\mathbf{F}_{k}$ as the rotated channel vector. That is, since $\mathbf{F}^{H}$ is the combination of the rotated channel vectors $\mathbf{f}_{i} \in \mathbb{C}^{t \times 1}$ for $i=1, \ldots, r$, $\mathbf{F}$ can be regarded as the multiple MISO channels.

For the second stage, controlled beamforming, which is implemented by applying MMSE QR decomposition to the combination of the rotated channels, is employed at the base station. As in the algorithm of [16] for MISO, the QR decomposition is obtained using the Gram-Schmidt orthogonalization procedure (e.g. to the rows of $\mathbf{F}$ in this case). Hence, the block MMSE QR decomposition consists of two consecutive procedures such that geometrical projection is performed to obtain $\mathbf{F}$ using SVD decomposition of $\mathbf{H}_{k}$ for all $k$ and then the finite dimensional subspace is determined by $\mathrm{QR}$ process with $\mathbf{F}$. Mathematically, MMSE QR decomposition of $\mathbf{F}$ leads to the solution for transmit beamforming satisfying the constraints in Theorem 1 . The solution is $\mathbf{W}_{\mathrm{tx}}=\left[\mathbf{w}_{1}, \mathbf{w}_{2}, \ldots, \mathbf{w}_{r}\right]$ where $\mathbf{w}_{j}$ for $j=1, \ldots, r$ is given by

$$
\mathbf{w}_{j}=\frac{\tilde{\mathbf{w}}_{j}}{\left\|\tilde{\mathbf{w}}_{j}\right\|}, \tilde{\mathbf{w}}_{j}=\left(\sum_{l=1}^{j-1} \mathbf{f}_{l} \mathbf{f}_{l}^{H}+\frac{r}{P} \mathbf{I}\right)^{-1} \mathbf{f}_{j}
$$

when equal power allocation and no ordering are assumed for different $\mathbf{f}_{l}$. The transmitted signal is represented as

$$
\mathbf{r}=\mathbf{F x}+\mathbf{z}=\mathbf{F W}_{\mathrm{tx}} \hat{\mathbf{s}}+\mathbf{z}
$$

where $\hat{\mathbf{s}}$ is the DPC encoded signal vector from the original signal vector $\mathbf{s}, \mathbf{z}=\left[\mathbf{z}_{1}^{T}, \ldots, \mathbf{z}_{k}^{T}\right]^{T}$ and $\mathbf{r}=\left[\mathbf{r}_{1}^{T}, \ldots, \mathbf{r}_{k}^{T}\right]^{T}$, in which $\mathbf{r}_{k}=\mathbf{U}_{k}^{H} \mathbf{y}_{k}$. The optimality of block QR decomposition and the actual implementation of this technique, i.e., determining the optimal strategy for power allocation and ordering, are treated in the following two subsections, respectively.

\subsection{Optimality of Block QR Decomposition}

In order to derive the procedure employing known interference cancellation, the congregate interfering channel matrix is defined as

$$
\overline{\mathbf{H}}_{k}=\left[\begin{array}{llll}
\mathbf{H}_{1}^{T} & \mathbf{H}_{2}^{T} & \cdots & \mathbf{H}_{k-1}^{T}
\end{array}\right]^{T} .
$$

Theorem 1: The objective of the transmit covariance matrix design for block MMSE-DP is to find a covariance matrix set that maximizes the system throughput, subject to the unknown-interference free constraint and the sum power constraint. Note that the unknown-interference free constraint leads to the block MMSE-DP solution, which reduces complexity and feedback overhead at the expense of negligible throughput performance degradation. The transmit covariance matrix satisfying this objective is obtained by $Q R$ decomposition of $\mathbf{F}$ with appropriate power allocation.

Proof: The problem may be formulated as finding a transmit covariance matrix set, $\left\{\boldsymbol{\Sigma}_{i}^{*}\right\}_{k=1}^{K}$, such that

$$
\left\{\boldsymbol{\Sigma}_{k}^{*}\right\}_{k=1}^{K}=\arg \max _{\left\{\boldsymbol{\Sigma}_{k}\right\}_{k=1}^{K}} R_{\mathrm{BM}}\left(\left\{\boldsymbol{\Sigma}_{k}\right\}_{k=1}^{K}\right)
$$

where

$R_{\mathrm{BM}}\left(\left\{\boldsymbol{\Sigma}_{k}\right\}_{k=1}^{K}\right)=\sum_{k=1}^{K} \log _{2} \frac{\left|\mathbf{I}+\mathbf{H}_{k}\left(\boldsymbol{\Sigma}_{k}+\cdots+\boldsymbol{\Sigma}_{K}\right) \mathbf{H}_{k}^{H}\right|}{\left|\mathbf{I}+\mathbf{H}_{k}\left(\boldsymbol{\Sigma}_{k+1}+\cdots+\boldsymbol{\Sigma}_{K}\right) \mathbf{H}_{k}^{H}\right|}$,

subject to the unknown-interference free constraint

$$
\mathbf{H}_{i} \boldsymbol{\Sigma}_{k}=\mathbf{F}_{i} \boldsymbol{\Sigma}_{k}=\mathbf{0}, k, i=1,2, \ldots, K, i<k
$$

and the sum power constraint

$$
\sum_{k=1}^{K} \operatorname{tr}\left(\boldsymbol{\Sigma}_{k}\right) \leq P, \mathbf{x}^{H} \boldsymbol{\Sigma}_{k} \mathbf{x} \geq 0, \forall \mathbf{x} \in \mathbb{C}^{t}, \forall k
$$

The constraint of (9) ensures that no interference to user $k$ is caused by the users from $k+1$ to $K$, noting that interference from other users (i.e. $1, \ldots, k-1$ ) is presubtracted by known interference cancellation (i.e. DPC encoding). Let $\overline{\mathbf{V}}_{k}^{0}$ consist of the right singular vectors corresponding to the zero singular values of $\overline{\mathbf{H}}_{k}$, which is equivalently obtained by SVD of $\overline{\mathbf{F}}_{k}=\left[\mathbf{F}_{1}^{H}, \mathbf{F}_{2}^{H}, \ldots, \mathbf{F}_{k-1}^{H}\right]^{H}$ as follows

$$
\overline{\mathbf{F}}_{k}=\left[\begin{array}{cc}
\overline{\mathbf{D}}_{k} & \mathbf{0} \\
\mathbf{0} & \mathbf{0}
\end{array}\right]\left[\begin{array}{cc}
\overline{\mathbf{V}}_{k}^{1} & \overline{\mathbf{V}}_{k}^{0}
\end{array}\right]^{H}
$$

where $\overline{\mathbf{D}}_{k}$ is a diagonal matrix. Thus, constructing $\boldsymbol{\Sigma}_{k}$ with $\overline{\mathbf{V}}_{k}^{0}$ satisfies the constraint in (9), i.e., $\boldsymbol{\Sigma}_{k}=\overline{\mathbf{V}}_{k}^{0} \overline{\boldsymbol{\Sigma}}_{k} \overline{\mathbf{V}}_{k}^{0 H}$ where $\overline{\boldsymbol{\Sigma}}_{k}$ is designed as follows. When the constraint in (9) is satisfied, the system throughput is simply derived as

$$
R_{\mathrm{BM}}^{*}=\max _{\left\{\boldsymbol{\Sigma}_{k}\right\}_{k=1}^{K}} R_{\mathrm{BM}}\left(\left\{\boldsymbol{\Sigma}_{k}\right\}_{k=1}^{K}\right)
$$

${ }^{\dagger}$ Each element of $\left\{\boldsymbol{\Sigma}_{k}^{*}\right\}_{k=1}^{K}$ is a positive semidefinite matrix, since it is a covariance matrix as defined in Sect. 2. 


$$
=\sum_{k=1}^{K} \max _{\overline{\boldsymbol{\Sigma}}_{k}} \log _{2}\left|\mathbf{I}+\mathbf{H}_{k} \overline{\mathbf{V}}_{k}^{0} \overline{\boldsymbol{\Sigma}}_{k} \overline{\mathbf{V}}_{k}^{0 H} \mathbf{H}_{k}^{H}\right|
$$

where $R_{\mathrm{BM}}^{*}$ is upper bounded by the sum capacity of the MIMO BC, $C_{\mathrm{BC}}$, while the equality between $R_{\mathrm{BM}}^{*}$ and $C_{\mathrm{BC}}$ holds if and only if $r_{k}=1$ for all $k$. The optimization of (12) is done by the SVD of $\mathbf{H}_{k} \overline{\mathbf{V}}_{k}^{0}=\tilde{\mathbf{U}}_{k} \tilde{\mathbf{D}}_{k} \tilde{\mathbf{V}}_{k}^{H}$, and hence

$$
\boldsymbol{\Sigma}_{k}^{*}=\overline{\mathbf{V}}_{k}^{0} \tilde{\mathbf{V}}_{k} \mathbf{P}_{k} \tilde{\mathbf{V}}_{k}^{H} \overline{\mathbf{V}}_{k}^{0 H} .
$$

where the diagonal matrix $\mathbf{P}_{k}$ is designed to satisfy the sum power constraint in (10). For example, optimal $\left\{\mathbf{P}_{k}\right\}_{k=1}^{K}$ can be found by performing SP-IWF with total multiple MISO channels $\mathbf{F}$. Moreover, the procedure described in (11)-(13) is mathematically equivalent to $\mathrm{QR}$ decomposition of $\mathbf{F}$, or equivalent to block QR decomposition of $\left\{\mathbf{H}_{k}\right\}_{k=1}^{K}$. When MMSE filtering is considered, MMSE QR decomposition [26] also can be used instead, which leads to the solution with $\mathbf{W}_{\mathrm{tx}}$ in (4) ${ }^{\dagger}$.

\subsection{Greedy Multi-Channel Selection Diversity}

Multiuser diversity is the promising solution to achieve the sum capacity of the multiuser channel. In the proposed scheme, we select the strong rotated channel vectors among available multi-user rotated channel vectors. Greedy MCSD is processed through the greedy-type ordering and selection of the channel vectors for active users. When channel vectors are selected and ordered, diversity gain is achieved with the increase of the number of users and antennas therein. The similar approach for MISO case was greedy ZF-DP as described in [16]. In our proposed method, we present two key functions. Firstly, the rotated channel vectors $\left\{\mathbf{f}_{i}\right\}_{i}$ are exploited instead of the channel vectors $\left\{\mathbf{h}_{i}\right\}_{i}$, since we consider multi-channel selection diversity but not just multiuser selection. Secondly, to further reduce the feedback overhead, a part of the channel selection process is performed at the user-side as well.

Let $A \subset\{1, \ldots, r\},|A| \leq \frac{1}{2} t(t+1)$ be a subset of the rotated channel vector indices that the base station selects for transmission using MCSD, and $\mathbf{F}(A)^{H}=\left[\mathbf{f}_{1}^{A}, \ldots, \mathbf{f}_{|A|}^{A}\right]$ be the corresponding submatrix of $\mathbf{F}$, where $\mathbf{f}_{l}^{A}$ is the $l$ th column vector of $\mathbf{F}(A)^{H}$. The $t \times|A|$ unitary beamforming matrix $\mathbf{W}_{\mathrm{tx}}(A)=\left[\mathbf{w}_{1}^{A}, \ldots, \mathbf{w}_{|A|}^{A}\right]$ in (5) now must be obtained by MMSE QR decomposition of submatrix $\mathbf{F}(A)$. The maximum sum-rate of this system is then given by

$$
\begin{aligned}
R_{\mathrm{MCSD}} & =\max _{A} f_{\mathrm{a}}(A) \\
& \leq R_{\mathrm{BM}}\left(\left\{\boldsymbol{\Sigma}_{i}^{*}\right\}_{i=1}^{K}\right) .
\end{aligned}
$$

The cost function $f_{\mathrm{a}}(A)$ is defined as

$$
\begin{aligned}
f_{\mathrm{a}}(A)= & \log _{2}\left|\frac{P}{|A|} \mathbf{\Phi}_{\mathrm{a},|A|}\right| \\
= & \log _{2}\left|\frac{P}{|A|} \boldsymbol{\Phi}_{\mathrm{a},|A|-1}\right|+ \\
& \log _{2}\left|\mathbf{I}+\mathbf{f}_{|A|}^{A H} \tilde{\mathbf{w}}_{|A|}^{A}\right|
\end{aligned}
$$

where $\boldsymbol{\Phi}_{\mathrm{a}, j}=\sum_{l=1}^{j} \mathbf{f}_{i}^{A} \mathbf{f}_{i}^{A H}+\frac{|A|}{P} \mathbf{I}$ and as in (4), $\mathbf{w}_{j}^{A}=\tilde{\mathbf{w}}_{j}^{A} /\left\|\tilde{\mathbf{w}}_{j}^{A}\right\|$, $\tilde{\mathbf{w}}_{j}^{A}=\boldsymbol{\Phi}_{\mathrm{a}, j-1}^{-1} \mathbf{f}_{j}^{A}$. The equality in (14) holds if and only if $|A|$, ordering, and power allocation of the selected channels are optimized, whereas power is allowed to be equally distributed among the selected rotated channels with $\frac{P}{|A|}$ in (14). Moreover, we also take into account the approach that each user selects and feeds back $L$ active channel vectors out of $\min \left(t, r_{k}\right)$ rotated channel vectors corresponding to the $L$ largest eigenmodes, where eigenmode $\lambda_{k, i}=$ $\left|d_{k, i}\right|^{2}$ is the power of the $i$ th diagonal element of $\mathbf{D}_{k}=$ $\operatorname{diag}\left(d_{k, 1}, \ldots, d_{k, \min \left(t, r_{k}\right)}\right)$. This approach offers further reduction the feedback amount by a factor of $\min \left(t, r_{k}\right) / L$. Note that although the feedback amount is significantly reduced by user-side selection, the loss of throughput performance in MCSD is negligible as analyzed in Theorem 3.

\section{Performance Analysis}

In this section, the performance analysis is presented. Throughout this paper, the entries of $\mathbf{H}_{k}$ are assumed to be i.i.d. zero-mean complex-Gaussian random variables.

Lemma 1: Assume that CSIT of all user $j(\neq k)$, i.e., $\left\{\mathbf{H}_{j}\right\}_{j \neq k}$, is not known to user $k$. That is, CSIT of all user $j$ is not delivered to user $k$ from the transmitter as well as not exchanged between users. In this case, each user $k$ can estimate the achievable throughout by using $\mathbf{F}_{k}=\mathbf{U}_{k}^{H} \mathbf{H}_{k}$, assuming that interference from all user $j$ is averaged out.

Proof: By applying the duality of MIMO BC and MAC [10], the sum capacity of an MIMO BC, which is equivalent to that of a dual MIMO MAC, with perfect channel knowledge at the transmitter is given by

$$
C_{\mathrm{BC}}=\max _{\sum_{i=1}^{K} \operatorname{tr}\left(\mathbf{S}_{i}\right) \leq P, \mathbf{S}_{i} \geq 0} f_{\mathrm{s}}\left(\left\{\mathbf{S}_{i}\right\}\right)
$$

where $\mathbf{S}_{i}$ is the transmit covariance matrix of user $i$ in a dual MIMO MAC. The cost function $f_{\mathrm{s}}\left(\left\{\mathbf{S}_{i}\right\}\right)$ is given by

$$
\begin{aligned}
f_{\mathbf{s}}\left(\left\{\mathbf{S}_{i}\right\}\right) & =\log _{2}\left|\mathbf{I}+\sum_{i=1}^{K} \mathbf{H}_{i}^{H} \mathbf{S}_{i} \mathbf{H}_{i}\right| \\
& =\log _{2}\left|\mathbf{\Phi}_{\mathbf{s}}\right|+\log _{2}\left|\mathbf{I}+\widetilde{\mathbf{H}}_{k}^{H} \mathbf{S}_{k} \widetilde{\mathbf{H}}_{k}\right|
\end{aligned}
$$

where $\widetilde{\mathbf{H}}_{k}=\mathbf{H}_{k} \boldsymbol{\Phi}_{\mathrm{s}}^{-1 / 2}$ and $\boldsymbol{\Phi}_{\mathrm{s}}=\mathbf{I}+\sum_{i \neq k} \mathbf{H}_{i}^{H} \mathbf{S}_{i} \mathbf{H}_{i}$. Because of the assumption that user $k$ is not allowed to know CSIT of all other users (except $E\left[\boldsymbol{\Phi}_{\mathrm{s}}\right]=\alpha \mathrm{I}$ where $\alpha=1+\frac{K-1}{K} \frac{P}{t}$ is a scalar), $\widetilde{\mathbf{H}}_{k}$ is transformed to $\mathbf{G}_{j}=\mathbf{H}_{k} E\left[\boldsymbol{\Phi}_{\mathrm{s}}\right]^{-1 / 2}=\frac{1}{\alpha} \mathbf{H}_{k}$. Hence, the maximum achievable rate can be derived by applying SVD to $\mathbf{G}_{j}$, where $\mathbf{H}_{k}$ is rotated by receive beamforming such that $\mathbf{F}_{k}=\mathbf{U}_{k}^{H} \mathbf{H}_{k}$.

Lemma 2: We compare the performance of block MMSEDP with (non-block) MMSE-DP in terms of the achievable throughput. In block MMSE-DP, receive beamforming is performed by the left unitary matrix of the corresponding

${ }^{\dagger}$ For example, the transmit covariance matrix of user $k$ in (4) is given by $\boldsymbol{\Sigma}_{k, \text { mmse }}^{*}=\mathbf{P}_{k} \mathbf{w}_{k} \mathbf{w}_{k}^{H}$ when $r_{k}=1$. 
channel, whereas MMSE-DP is known to be optimal for non-cooperative reception across receive antennas. For average throughput, block MMSE-DP outperforms MMSEDP in an MIMO BC.

Proof: The problem in question can be seen as the comparison between two different scenarios: beamforming with $\mathbf{W}_{\mathrm{rx}}=\mathbf{U}_{k}^{H}$ and beamforming with $\mathbf{W}_{\mathrm{rx}}=\mathbf{I}$. Since SVDbased processing is optimal for the single-user case, the capacity of the dual MAC subject to $\mathbf{W}_{\mathrm{rx}}=\mathbf{U}_{k}^{H}$ is larger or equal to that subject to $\mathbf{W}_{\mathrm{rx}}=\mathbf{I}$, as follows

$$
\max _{\boldsymbol{\Lambda}} \log _{2}\left|\mathbf{I}+\mathbf{R}_{\mathrm{U}, k}(\mathbf{\Lambda})\right| \geq \max _{\boldsymbol{\Lambda}} \log _{2}\left|\mathbf{I}+\mathbf{R}_{\mathrm{I}, k}(\boldsymbol{\Lambda})\right|
$$

where $\mathbf{R}_{\mathrm{U}, k}(\boldsymbol{\Lambda})=\mathbf{H}_{k}^{H} \mathbf{U}_{k} \boldsymbol{\Lambda} \mathbf{U}_{k}^{H} \mathbf{H}_{k}=\mathbf{F}_{k}^{H} \boldsymbol{\Lambda} \mathbf{F}_{k}, \mathbf{R}_{\mathrm{I}, k}(\boldsymbol{\Lambda})=$ $\mathbf{H}_{k}^{H} \boldsymbol{\Lambda} \mathbf{H}_{k}$, and $\boldsymbol{\Lambda}$ is constrained to be a diagonal matrix. In the following, we show that the single-user inequality in (18) leads to the proof of the given Lemma. Applying the duality principle as in (16), the sum capacity obtained based on non-cooperative reception in the $k$ th user, i.e., $\mathbf{W}_{\mathrm{rx}}=\mathbf{I}$, can be represented as

$$
R_{\mathrm{I}}=\max _{\boldsymbol{\Lambda}, \mathbf{Q}_{i}} \log _{2}\left|\mathbf{I}+\mathbf{R}_{\mathrm{I}, k}(\mathbf{\Lambda})+\sum_{i \neq k} \mathbf{H}_{i}^{H} \mathbf{Q}_{i} \mathbf{H}_{i}\right|
$$

where the maximization is subject to $\operatorname{tr}(\boldsymbol{\Lambda})+\sum_{i \neq k} \operatorname{tr}\left(\mathbf{Q}_{i}\right) \leq P$ and $\Lambda, \mathbf{Q}_{i} \geq 0$. Similarly, the maximum sum-rate obtained with the unitary beamforming at the receiver is expressed as

$$
\boldsymbol{R}_{\mathrm{U}}=\max _{\boldsymbol{\Lambda}, \mathbf{Q}_{i}} \log _{2}\left|\mathbf{I}+\mathbf{R}_{\mathrm{U}, k}(\boldsymbol{\Lambda})+\sum_{i \neq k} \mathbf{H}_{i}^{H} \mathbf{Q}_{i} \mathbf{H}_{i}\right| \text {. }
$$

Observing the inequality in (18) and the same term added in both (19) and (21), we see that the average maximum sumrate $E\left[R_{\mathrm{U}}\right]$ is larger or equal to the average sum capacity for non-cooperative reception $E\left[R_{\mathrm{I}}\right]$. Applying this result to the problem, it follows that

$$
E\left[R_{\mathrm{BM}}^{*}\right] \geq E\left[R_{\mathrm{MMSE}-\mathrm{DP}}\right]
$$

which completes the proof. Note that it might not be true for instantaneous throughput.

Theorem 2: In MIMO BC, the system with beamforming at each receiver using the left singular matrix offers the average throughput that is no worse than using any fixed unitary matrix beam at all receivers.

Proof: The proof is easy. We prove Theorem 2 based on Lemmas 1 and 2. In terms of minimizing interference, the fixed beam scheme performs better than the proposed beamforming in certain channel realizations or vice versa. Both cases are equally probable, i.e., $p_{1}=p_{2}$ where $p_{1}$ and $p_{2}$ represent the probability of each case, respectively. This follows from the fact that $\left\{\mathbf{H}_{k}\right\}_{k}$ is i.i.d. over index $k$. In other realizations with probability $p_{3}=1-\left(p_{1}+p_{2}\right)$, the proposed scheme always outperforms the other because of the signal-to-noise ratio (SNR) advantage. Thus, the average performance of the fixed beam scheme is no better than the proposed scheme as derived in (18). Note that the equality holds only when $p_{3}=0$.
Theorem 3: In the proposed MCSD scheme, we select the strong effective (sub) channels, of which the number is larger or equal to one. The sum-rate achievable with the selected channels is almost equal to the sum capacity of total multiple MISO channels $\left\{\mathbf{F}_{k}\right\}_{k}$ in (3).

Proof: This channel selection exploits the fact that similar eigen-vectors, or sub channels, can exist in multiple MISO channels. Since the eigen-vectors inside a single user MIMO channel are not similar to one another, i.e, in fact they are orthogonal, the problem in this theorem is different from the water-filling problem in single-user MIMO. Hence, we prove Theorem 3 by showing that in the multiple MISO channels, optimal power allocation is obtained based on solely the maximum eigen-values of each group if eigenvectors in each group are nearly the same. Let $\left\{v_{l, m}\right\}_{m}=\left\{\lambda_{i}\right\}_{i}$ if $\left\|\overline{\mathbf{v}}_{l}-\mathbf{v}_{i}\right\|^{2} \leq\left\|\overline{\mathbf{v}}_{n}-\mathbf{v}_{i}\right\|^{2}$ for all $n \neq l, l=1, \ldots, L_{e}$, where $\lambda_{i}$ and $\mathbf{v}_{i}$ are the $i$ th eigen-value and the $i$ th eigen-vector, respectively. We also assume that $L_{e}$ is large enough so that $\left\|\overline{\mathbf{v}}_{l}-\mathbf{v}_{i}\right\|^{2}$ is sufficiently small for all $l$. The sum capacity is then given by

$$
\begin{aligned}
& R_{\mathrm{BM}}\left(\left\{\Sigma_{i}^{*}\right\}_{i=1}^{K}\right) \\
& =\max _{P_{i} \geq 0, \sum_{i} P_{i} \leq P} \log _{2}\left|\mathbf{I}+\sum_{i=1}^{\bar{r}} P_{i} \lambda_{i} \mathbf{v}_{i} \mathbf{v}_{i}^{H}\right| \\
& \approx \max _{P_{l, m} \geq 0, \Sigma_{l, m} P_{l, m} \leq P} \log _{2}\left|\mathbf{I}+\sum_{l} \sum_{m} P_{l, m} v_{l, m} \overline{\mathbf{v}}_{l} \overline{\mathbf{v}}_{l}^{H}\right| \\
& =\max _{P_{l} \geq 0, \Sigma_{l} P_{l} \leq P} \log _{2}\left|\mathbf{I}+\sum_{l} P_{l} v_{l, \max } \overline{\mathbf{v}}_{l} \overline{\mathbf{v}}_{l}^{H}\right|
\end{aligned}
$$

where $\bar{r}=\sum_{k=1}^{K} \min \left(t, r_{k}\right)$, and $v_{l, \max }=\max _{m}\left\{v_{l, m}\right\}$. In (22), we use the property of multiuser diversity [27], in which the sum rate is maximized by allocating no transmit power to certain sub channels if there is any other sub channel with the same direction and higher gain.

\section{Numerical Results}

In this section, numerical results are presented. In Figs. 3 and 4, we compare the ergodic sum-rate performance of different MIMO downlink strategies. The SNR is assumed to be $10 \mathrm{~dB}$. Given the number of users, TDMAMIMO achieves the maximum sum-rate corresponding to the largest single-user capacity, which shows relatively a small gain in proportion to the number of users. When the number of the active channel vectors is equal to the number of the rotated channel vectors with the number of users being one, the performance of the proposed novel scheme is the same as that of TDMA-MIMO since in both cases receivers feed back the rotated channel matrix $\mathbf{F}_{k}=\mathbf{D}_{k} \mathbf{V}_{k}^{H}$, instead of the full channel matrix $\mathbf{H}_{k}$. In both figures, the performance of the novel scheme with full rotated channel vectors (i.e. $L=2,4$, respectively) is extremely close to the sum capacity driven by SP-IWF, while the performance with the active (partial rotated) channel vector (i.e. $L=1$ ) 


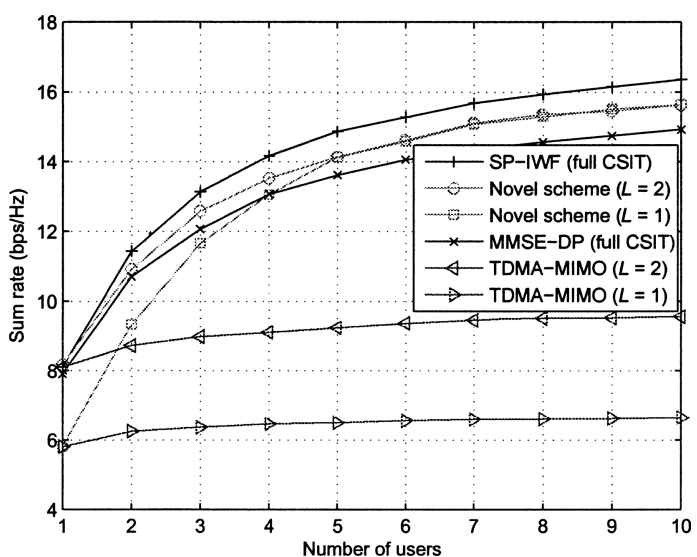

Fig. 3 Ergodic sum-rate comparison when $t=4$ and $r_{k}=2$ for all $k$.

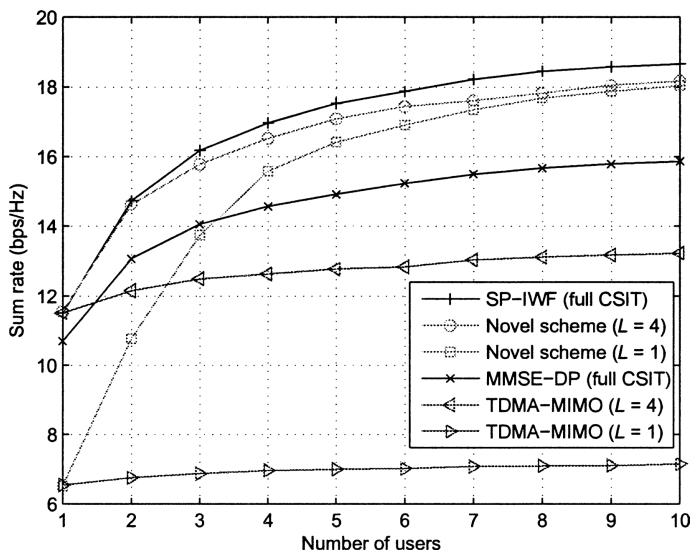

Fig. 4 Ergodic sum-rate comparison when $t=4$ and $r_{k}=4$ for all $k$.

reaches the sum-rate with full vectors when the number of users is equal to 5 and 10 , respectively. Both figures show sum-rate improvement of $2 \mathrm{bps} / \mathrm{Hz}$ over MMSE-DP scheme with full channel feedback and the gap of $0.4 \mathrm{bps} / \mathrm{Hz}$ from SP-IWF.

Furthermore, both figures illustrate the behaviors of sum-rate corresponding to different feedback overheads. In Fig. 3, each user has two eigenmodes, i.e., two rotated channel vectors, available since four transmit and two receive antennas are assumed. The sum-rate of the novel scheme with feedback of one active channel vector (one eigenvector multiplied by the corresponding eigenvalue that is the largest one) gets tightly close to the performance having feedback of two active channel vectors when the number of users is five. Contrastingly, TDMA-MIMO with one vector never becomes close to TDMA-MIMO with two vector. Four transmit and four receive antennas are considered in Fig. 4, where two feedback signaling (i.e. one, four active channel vectors) are examined for the novel and TDMAMIMO schemes. Both figures show that the novel scheme with reduced feedback, i.e., with the fewer active channel vectors, achieves slightly lower rate performance with small number of users compared to the scheme with full rotated channel vector, and however, the performance ap- proaches extremely close to the upper bound as the number of users increases. Note that when the feedback bandwidth is fixed, the reduction of active channel vectors may improve the quality of feedback signaling, of which the level can be represented based on the Shannon distortion-rate function $D_{k}(R) \propto 2^{-2 \alpha_{k} R}$ where $R$ is the given feedback rate and $\alpha_{k}=L / \min \left(t, r_{k}\right)$ [21]. Therefore, in the proposed scheme feedback of active channel vectors is shown to have the equivalent sum-rate performance with feedback of full rotated channel vectors, resulting in the outstanding feedback robustness. That is, the feedback signaling per user can be significantly reduced with the increase of the number of users.

\section{Reconfigurable Implementation}

Wireless MIMO systems would be capable of operating in a wide variety of scenarios with respect to the associated parameters, e.g., the number of users and the degrees of knowledge of CSIT [28]. Reconfigurability, namely adaptability, is an important design principle that can be considered for MIMO systems in a diverse environment. Software defined radio, as an example of reconfigurable systems, attempts to achieve the best performance in each respective case whereas systems without reconfigurability maintain reasonable performance, which is considered to be robust in a varying environment. The study of adaptability in MIMO systems is motivated on a fundamental tradeoff between the number of active eigenmodes and the required feedback rate as discussed in Sect. 6. The reconfigurable system chooses the best number of active eigenmodes in terms of throughput performance given a constraint on the required amount of feedback. Although the TDMA-MIMO performance strongly depends on the trade-off, reconfigurability with respect to the number of users offers almost no performance benefit for the TDMA-MIMO system as shown in Fig. 4. However, a significant performance advantage is observed for greedy MCSD with block MMSE-DP.

Figure 5 compares a reconfigurable system against a robust system with the predetermined parameters in terms of sum rate. It is assumed that $t=r_{k}=4$ for all $k$. Based on the aforementioned MIMO fundamental trade-off, the reconfigurable MCSD operates with the variable number of active vectors: $L=4$ for $K=1, L=2$ for $K=2$, and $L=1$ for $K=4$. Note that the total amount of feedback stays the same for all three cases, i.e. $K L=4$. For robust systems, however, we consider MCSD with the fixed number of active vectors for all $K$, e.g., $L=1,2,4$, since it does not allow operating with variable parameters. Thus, the required amount of feedback increases by the number of active vectors as a single user is added to the system. The figure represents that the reconfigurable system has the throughput performance two times higher than that of the robust system with $L=1$ at $K=1$, since it selects $L=4$ for $K=1$. On the other hand, the reconfigurable system chooses $L=1$ for $K=4$ and decreases the required feedback rate four times lower than that of the robust system with $L=4$ and $K=4$. 


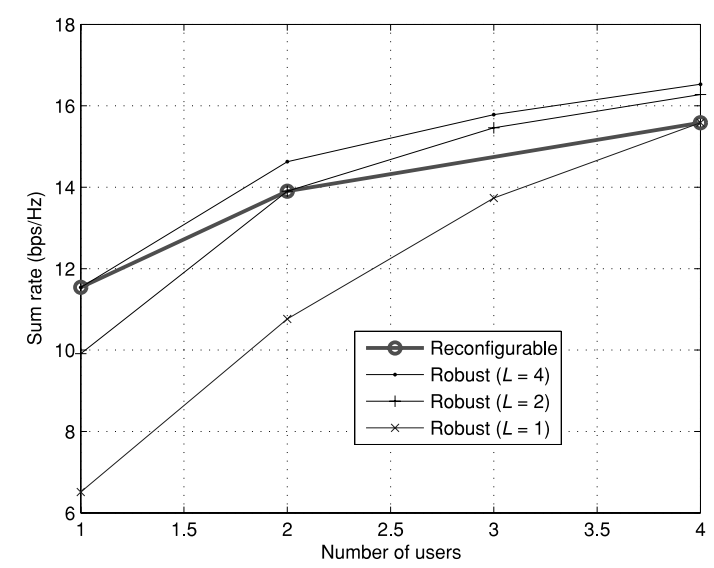

Fig. 5 Reconfigurability to the number of users when $t=4$ and $r_{k}=4$ for all $k$.

Hence, we can see that the reconfigurable system can reduce a considerable amount of feedback by adaptively selecting the number of active vectors without losing noticeable performance. The figure also shows that the throughput of the reconfigurable system asymptotically converges on that of the robust system with the highest performance as the number of users increases.

\section{Conclusion}

In this paper, we have proposed a multiuser MIMO transmission scheme that is efficient in terms of computational complexity and feedback overhead while obtaining near the maximum sum-rate of BC. Our novel scheme has employed block MMSE-DP at the transmitter, which reduces the computational complexity of designing transmit covariance matrices. Using MCSD in combination with block MMSE-DP, the proposed scheme with partial CSIT has still achieved the near-optimal sum capacity, which was not observed in TDMA-MIMO. Numerical results have shown that the gain of sum-rate is $2 \mathrm{bps} / \mathrm{Hz}$ over the conventional MMSE-DP scheme with full channel feedback and the gap from SPIWF is negligibly small (i.e. $0.4 \mathrm{bps} / \mathrm{Hz}$ ). Moreover, the proposed scheme has been shown to be appropriate for reconfigurable implementation and to reduce a considerable amount of feedback by adaptively selecting the number of active vectors without losing noticeable performance.

\section{Acknowledgments}

The authors wish to express their gratitude to Haewoon Nam in the University of Texas at Austin and Dr. Jianjun $\mathrm{Li}$ in Samsung Advanced Institute of Technology (SAIT) for valuable comments. This work was supported in part by National Research Laboratory (NRL) Program.

\section{References}

[1] J.S. Kim, H. Kim, and K.B. Lee, "Limited feedback signaling for MIMO broadcast channels," 6th IEEE Workshop on Signal Processing Advances in Wireless Communications (SPAWC), pp.855-859,
New York, June 2005.

[2] G.J. Foschini, "Layered space-time architecture for wireless communication in a fading environment when using multi-element antennas," Bell Labs Tech. J., vol.1, no.2, pp.41-59, 1996.

[3] I.E. Telatar, "Capacity of multi-antenna Gaussian channels," Europ. Trans. Telecommun., vol.10, no.6, pp.585-596, Nov. 1999.

[4] A.J. Paulraj and T. Kailath, "Increasing capacity in wireless broadcast systems using distributed transmission/directional reception (DTDR)." US Patent no.5,345,599, 1993.

[5] D. Gore and A.J. Paulraj, "Optimal antenna selection in MIMO systems with space-time block coding," IEICE Trans. Commun., vol.E84-B, no.7, pp.1713-1719, July 2001.

[6] R.W. Heath, Jr. and A.J. Paulraj, "Capacity maximizing linear spacetime codes," IEICE Trans. Electron., vol.E85-C, no.3, pp.428-435, March 2002.

[7] H. Sato, "An outer bound on the capacity region of the broadcast channel," IEEE Trans. Inf. Theory, vol.24, pp.374-3778, May 1978.

[8] G. Caire and S. Shamai, "On the achievable throughput of a multiantenna Gaussian broadcast channel," IEEE Trans. Inf. Theory, vol.49, no.7, pp.1691-1706, July 2003.

[9] H. Viswanathan, S. Venkatesan, and H.C. Huang, "Downlink capacity evaluation of cellular networks with known interference cancellation,” IEEE J. Sel. Areas Commun., vol.21, no.5, pp.802-811, June 2003

[10] S. Vishwanath, N. Jindal, and A. Goldsmith, "Duality, achievable rates, and sum-rate capacity of MIMO broadcast channels," IEEE Trans. Inf. Theory, vol.49, no.10, pp.2658-2668, Oct. 2003.

[11] P. Viswanath and D.N. Tse, "Sum capacity of the vector Gaussian broadcast channel and uplink-downlink duality," IEEE Trans. Inf. Theory, vol.49, no.8, pp.1912-1921, Aug. 2003.

[12] W. Yu and J.M. Cioffi, "Sum capacity of Gaussian vector broadcast channels," IEEE Trans. Inf. Theory, vol.50, no.9, pp.1875-1892, Sept. 2004.

[13] N. Jindal, W. Rhee, S. Vishwanath, S. Jafar, and A. Goldsmith, "Sum power iterative water-filling for multi-antenna Gaussian broadcast channels," IEEE Trans. Inf. Theory, vol.51, no.4, pp.1570-1580, April 2005.

[14] M. Costa, "Writing on dirty paper," IEEE Trans. Inf. Theory, vol.29, no.3, pp.439-441, May 1983.

[15] W. Yu, W. Rhee, S. Boyd, and J.M. Cioffi, "Iterative water-filling for Gaussian vector multiple access channels," IEEE Trans. Inf. Theory, vol.50, no.1, pp.145-151, Jan. 2004.

[16] Z. Tu and R.S. Blum, "Multiuser diversity for a dirty paper approach," IEEE Commun. Lett., vol.7, no.8, pp.370-372, Aug. 2003.

[17] R.L. Choi and R.D. Murch, "A transmit processing technique for multiuser MIMO systems using a decomposition approach,” IEEE Trans. Wirel. Commun., vol.3, no.1, pp.20-24, Jan. 2004.

[18] Q.H. Spence, A.L. Swindlehurst, and M. Haardt, "Zero-forcing methods for downlink spatial multiplexing in multi-user MIMO channels,” IEEE Trans. Signal Process., vol.52, no.2, pp.461-471, Feb. 2004.

[19] P. Viswanath, D.N. Tse, and R. Laroia, "Opportunistic beamforming using dump antennas," IEEE Trans. Inf. Theory, vol.48, no.6, pp.1277-1294, June 2002.

[20] M. Sharif and B. Hassibi, "On the capacity of MIMO broadcast channels with partial side information," IEEE Trans. Inf. Theory, vol.51, no.2, pp.506-522, Feb. 2005.

[21] A. Gersho and R.M. Gray, Vector Quantization and Signal Compression, Kluwer Academic Press, 1992.

[22] D.J. Love, R.W. Heath, Jr., W. Santipach, and M.L. Honig, "What is the value of limited feedback for MIMO channels?," IEEE Commun. Mag., vol.42, no.10, pp.54-59, Oct. 2004.

[23] N. Jindal, W. Rhee, S. Vishwanath, S. Jafar, and A. Goldsmith, "DPC vs. TDMA for MIMO broadcast channels," IEEE Trans. Inf Theory, vol.51, no.5, pp.1783-1794, May 2005.

[24] J.S. Kim, H.J. Kim, C.S. Park, and K.B. Lee, "Space-time technique for wireless multiuser MIMO systems with SIC receivers," Proc. 
IEEE PIMRC, pp.2013-2017, Barcelona, Spain, Sept. 2004.

[25] D.J. Love, R.W. Heath, Jr., and T. Strohmer, "Grassmannian beamforming for multiple-input multiple-output wireless systems," IEEE Trans. Inf. Theory, vol.49, no.10, pp.2735-2747, Oct. 2003.

[26] R. Böhnke, D. Wübben, V. Kühn, and K.D. Kammeyer, "Reduced complexity MMSE detection for BLAST architectures," Proc. IEEE GLOBECOM, pp.2258-2262, San Francisco, CA, Dec. 2003.

[27] R. Knopp and P.A. Humblet, "Information capacity and power control in single-cell multiuser communications," Proc. IEEE ICC, pp.331-335, June 1995.

[28] M. Haardt and A. Alexiou, "Smart antennas and related technologies." WWRF-WG4 White Paper, May 2005.

[29] W. Yu, "Spatial multiplex in downlink multiuser multiple-antenna wireless environments," Proc. IEEE GLOBECOM, pp.1887-1891, Dec. 2003.

[30] W. Rhee, W. Yu, and J.M. Cioffi, "The optimality of beamforming in uplink multiuser wireless systems," IEEE Trans. Wirel. Commun., vol.3, no.1, pp.86-96, Jan. 2004.

[31] M. Airy, A. Forenza, R.W. Heath, Jr., and S. Shakkottai, "Practical Costa precoding for the multiple antenna broadcast channel," Proc. IEEE GLOBECOM, pp.3942-3946, Dallas, TX, Nov. 2004.

\section{Appendix: Greedy MMSE-DP Algorithm}

The algorithm for greedy MMSE-DP is outlined as follows:

1. The base station selects the index pairs of user and its receive antenna, $\left\{k_{j}, i_{j}\right\}_{j}, j \leq \frac{1}{2} t(t+1), j \leq r$ [29], [30] corresponding to the downlink channel matrices $\left\{\mathbf{H}_{k}\right\}_{k=1}^{K}$ where $\mathbf{H}_{k}^{H}=\left[\mathbf{h}_{k, 1}, \ldots, \mathbf{h}_{k, r_{k}}\right]$. Before starting the procedure, we take as initial value $m=0$.

2. Let $m=m+1$, and if $m>K$, stop the procedure. Otherwise, calculate the received SNR as function of $m$ for all $(k, i)$ as follows

$$
\gamma_{k, i}(m)=\mathbf{h}_{k, i}^{H}\left(\sum_{j=1}^{m-1} \mathbf{h}_{k_{j}, i_{j}} \mathbf{h}_{k_{j}, i_{j}}^{H}+\frac{m}{P} \mathbf{I}\right)^{-1} \mathbf{h}_{k, i},
$$

determine the $m$ th selected index pair of user and its antenna as follows

$$
\left(k_{m}, i_{m}\right)=\arg \max _{k \in 1, \ldots, K, i \in 1, \ldots, r_{k}} \gamma_{k, i}(m),
$$

and calculate the sum-rate achievable with $\gamma_{k_{1}, i_{1}}, \ldots$, $\gamma_{k_{m}, i_{m}}$ as follows

$$
R_{m}=\sum_{j=1}^{m} \log _{2}\left(1+\gamma_{k_{j}, i_{j}}(m)\right) .
$$

3. Repeat Step 2 until $R_{m} \leq R_{m-1}$.

4. Transmit

$$
\mathbf{x}=\frac{P}{m_{o}} \mathbf{W}_{\mathrm{tx}} \hat{\mathbf{s}}
$$

where $m_{o}=m-1, \hat{\mathbf{s}}=\left[\hat{s}_{k_{1}}, \hat{s}_{k_{2}}, \ldots, \hat{s}_{k_{m_{o}}}\right]^{T}$, and $\mathbf{W}_{\mathrm{tx}}=$ $\left[\mathbf{w}_{1}, \mathbf{w}_{2}, \ldots, \mathbf{w}_{m_{o}}\right]$. The transmit basis $\mathbf{w}_{j}$ is given by

$$
\mathbf{w}_{j}=\frac{\tilde{\mathbf{w}}_{j}}{\left\|\tilde{\mathbf{w}}_{j}\right\|}, \quad \tilde{\mathbf{w}}_{j}=\left(\sum_{l=1}^{j-1} \mathbf{h}_{k_{l}, i_{l}} \mathbf{h}_{k_{l}, i_{l}}^{H}+\frac{m_{o}}{P} \mathbf{I}\right)^{-1} \mathbf{h}_{k_{j}, i_{j}} .
$$

Here, $\hat{s}_{k_{j}}$ is the output of the DPC encoding equation parameterized as a function of $\left\{s_{k_{l}}, \mathbf{h}_{k_{j}, i_{j}}^{T} \mathbf{w}_{l}\right\}_{l=j}^{m_{o}}$, where the signal $s_{k_{l}}$ is associated to the $k_{l}$ th user [31]. Note that the total throughput of this scheme is given by $R_{\mathrm{MMSE}-\mathrm{DP}}=R_{m-1}$.

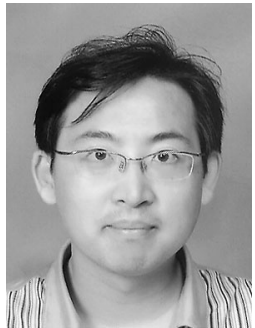

James (Sungjin) Kim was born in Korea in 1969. He obtained his Bachelor and Master of Engineering degree in Electronics and Communications Engineering from the College of Engineering, Hanyang University, Korea in 1994 and in 2000 , respectively. He is now pursuing his Doctor of Philosophy in Electrical and Computer Engineering from the College of Engineering, Seoul National University. In February 1994 he joined Samsung Advanced Institute of Technology, and he is now a senior member of technical research staff. Since 1999, he has been the Editor-in-Chief of 3GPP (WCDMA standard) Transmit Diversity TR. His research interests include the areas of transmit diversity (TxD), multiple-input and multipleoutput (MIMO), wireless scheduling and adaptive signal processing for $3 \mathrm{G}+/ 4 \mathrm{G}$ wireless communications.

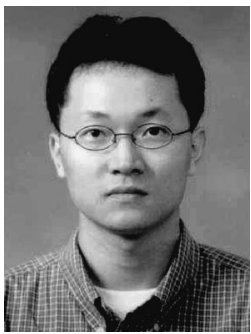

Hojin Kim was born in Korea in 1973. He obtained his Bachelor of Science in Electrical and Computer Engineering from Purdue University, Indiana in 1997. He received his Master of Science from the Electrical and Computer Engineering at the University of Florida, Florida in 2000. In 2000, he was with LG electronics institute of technology as a research engineer. Since 2001, he has been a research engineer at Samsung advanced institute of technology. His research interests include MIMO, OFDM, Ad-hoc network, and 3GPP standardization.

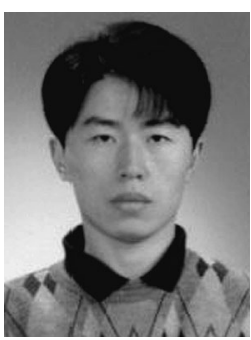

Chang Soon Park received the B.A.Sc and M.Eng. degrees from the School of Electrical Engineering and Computer Science, Seoul National University, Seoul, Korea, in 2000 and 2002 , respectively. He is now pursuing his Doctor of Philosophy in the School of Electrical Engineering and Computer Science, Seoul National University. 


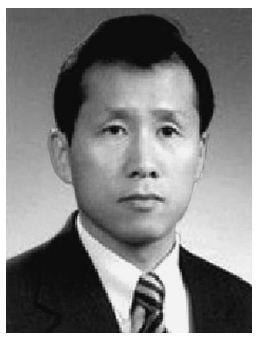

Kwang Bok Lee received the B.A.Sc. and M.Eng. degrees from the University of Toronto, Toronto, Ont., Canada, in 1982 and 1986, respectively, and the Ph.D. degree from McMaster University, Canada in 1990 . He was with Motorola Canada from 1982 to 1985, and Motorola USA from 1990 to 1996 as a Senior Staff Engineer. At Motorola, he was involved in the research and development of wireless communication systems. He was with Bell-Northern Research, Canada, from 1989 to 1990 . In March 1996, he joined the School of Electrical Engineering, Seoul National University, Seoul, Korea. Currently he is an Associate Professor in the School of Electrical Engineering. He was a Vice Chair of the School of Electrical Engineering from 2000 to 2002. He has been serving as a Consultant to a number of wireless industries. His research interests include mobile communications, communication technique covering physical layer and upper layer. He holds ten U.S. patents and seven Korean patents, and has a number of patents pending. Dr. Lee was an Editor of the IEEE Journal on Selected Areas in Communications, Wireless Series in 2001, and has been an Editor of the IEEE Transactions on Wireless Communications since 2002. And he was a co-chair of the ICC2005 Wireless Communication Symposium. He received the Best Paper Award from CDMA International Conference 2000 (CIC 2000), and the Best Teacher Award in 2003 from College of Engineering, Seoul National University. 